\title{
Genetic variants in the upstream region of activin receptor IIA are associated with female fertility in Japanese Black cattle
}

Shinji Sasaki ${ }^{*}$, Takayuki Ibi ${ }^{2}$, Tamako Matsuhashi ${ }^{3}$, Kenji Takeda ${ }^{3}$, Shogo Ikeda ${ }^{4}$, Mayumi Sugimoto ${ }^{1}$ and Yoshikazu Sugimoto ${ }^{5}$

\begin{abstract}
Background: Female fertility, a fundamental trait required for animal reproduction, has gradually declined in the last 2 decades in Japanese Black cattle. To identify associated genetic variants in Japanese Black cattle, we evaluated female fertility as a metric to describe the average inverse of the number of artificial inseminations required for conception from the first through the fourth parity $\left(\mathrm{ANAl}_{4}\right)$ and conducted a genome-wide association study (GWAS) using 430 animals with extreme $\mathrm{ANAl}_{4}$ values from 10,399 animals.

Results: We found that 2 variants, namely a single-nucleotide polymorphisms (SNP; g.48476925C > T) and a 3-bp indel (g.48476943_48476946insGGC), in the upstream region of the activin receptor IIA gene (ACVR2A) were associated with $\mathrm{ANAl}_{4}$. ACVR2A transcripts from Japanese Black cattle of the $Q$ haplotype, defined by the SNP and the 3-bp indel, with increased $\mathrm{ANAl}_{4}$ were 1.29-1.32-fold more abundant than q-derived transcripts. In agreement, reporter assay results revealed that the activity of the ACVR2A promoter was higher in reporter constructs with the $Q$ haplotype than in those with the $q$ haplotype by approximately 1.2 fold. Expression of exogenous ACVR2A induced dose-dependent increases of reporter activity from the follicle-stimulating hormone, beta polypeptide (FSHB) promoter in response to activin A in a pituitary gonadotrophic cell line. The findings suggested that sequence variations in the upstream region of ACVR2A with the $Q$ haplotype increased ACVR2A transcription, which in turn induced FSHB expression. This association was replicated using a sample population size of 1,433 animals; the frequency of the $Q$ haplotype was 0.39 , and $Q$-to- $q$ haplotype substitution resulted in an increase of 0.02 in terms of $\mathrm{ANAl}_{4}$.
\end{abstract}

Conclusions: This GWAS identified variants in the upstream region of ACVR2A, which were associated with female fertility in Japanese Black cattle. The variants affected the level of ACVR2A mRNA expression, which could lead to an allelic imbalance. This association was replicated with a sample population of 1,433 animals. Thus, the results suggest that the $Q$ haplotype could serve as a useful marker to select Japanese Black cattle with superior female fertility.

Keywords: Female fertility, Reproductive efficiency, Genome-wide association study, Activin receptor IIA (ACVR2A), Japanese Black cattle, Beef cattle

\footnotetext{
*Correspondence: sasakis@siag.or.jp

${ }^{1}$ National Livestock Breeding Center, Odakura, Nishigo, Fukushima 961-8511, Japan

Full list of author information is available at the end of the article
} 


\section{Background}

Fertile female cattle show clear estrous in a timely manner and become pregnant within a minimum number of artificial inseminations (AIs). However, over the past 2 decades, female fertility in AI breeding programs has been gradually declining in Japanese Black cattle; e.g., the first-AI conception rate decreased from $67.4 \%$ to 56 \% between 1992 and 2012 in Japan [1]. The trend has been also observed in dairy cattle $[1,2]$. Additional AIs increase costs related to semen, hormonal treatments, and AI technician fees, as well as feeding until the next AI. Therefore, farmers and breeders pay close attention to genetic factors related to improving female fertility for greater reproductive performance and profitability.

Recently, using a high-density single-nucleotide polymorphism (SNP) array [3, 4], genome-wide association studies (GWAS) have enabled researchers to scan the entire genome for related genetic factors and have identified a quantitative trait locus (QTL) for fertilityrelated traits in various cattle breeds [5-13] (reviewed in $[14,15])$; however, QTLs for fertility-related traits in Japanese Black cattle have remained fully unknown.

Japanese Black cattle are highly valued owing to the abundant marbling of meat caused by intramuscular fat depositions [16]. Strict selection for marbling under a closed breeding system in Japan [17] has made Japanese Black cattle genetically distinct from European cattle breeds [18]. Therefore, genome-wide QTL screening for female fertility needs to be applied to this breed.

The genetic parameters for calving interval-related traits have been evaluated until animals reach approximately 4 to 5 years of age in Japanese Black cattle in Japan [19]. Thus, the AI records of 10,399 animals at each parity, from the first through the third or fourth parity (see Methods section) were available. Multiple records from single animals are important for accurately evaluating fertility performances. However, the number of cows decreases as the age of cows increases, reflecting the culling of animals with lower fertility. Thus, in this study, to identify variants associated with female fertility in Japanese Black cattle, we evaluated female fertility as a metric to describe the average inverse of the number of AIs required for conception [20] from first through fourth parity $\left(\mathrm{ANAI}_{4}\right)$ and conducted a GWAS for this trait. The current GWAS identified associated variants in the upstream region of the activin receptor IIA gene $(A C V R 2 A)$, which serve as a key regulator of follicular growth in the ovaries by controlling folliclestimulating hormone $(F S H)$ expression.

\section{Results and discussion}

A QTL for $\mathrm{ANAl}_{4}$ was identified on bovine chromosome 2 (BTA2) in Japanese Black cattle

The heritability of $\mathrm{ANAI}_{4}$ was estimated to be 0.02 using the numerator-relationship matrix among 10,399 animals based on pedigree information, consistent with previous studies reporting the inverse of the number of inseminations required for conception [20] and female conceptionrelated traits in cattle (reviewed in $[15,21]$ ). As shown in Additional file 1, the distribution was sufficiently wide to discriminate between the upper- and lower-performance groups. Selective genotyping using animals with phenotypic values that deviate from the population mean is an effective method for reducing the sample sizes required to detect common SNPs associated with traits [22]. We selected 256 cows from the upper extreme $\left(85^{\text {th }}\right.$ percentile, average $\mathrm{ANAI}_{4}$ was 0.927 ) of the distribution and 174 cows from the lower extreme $\left(15^{\text {th }}\right.$ percentile, average $\mathrm{ANAI}_{4}$ is 0.399) among 10,399 cows with $\mathrm{ANAI}_{4}$ records. These samples were genotyped using the BovineSNP50K BeadChip, comprising probes for 54,001 SNPs. A total of 33,303 autosomal SNPs that passed our quality control criteria (call rate $>99 \%$, minor allele frequency $>0.01$, Hardy-Weinberg equilibrium $P>0.001$, and inclusion of the SNP on the BovineHD BeadChip) [23] were used for the association study. Analysis was performed using EMMAX software [24], which is based on a linear-mixed model approach using a genetic-relationship matrix estimated by SNP genotypes to model the correlation between the phenotypes of the sample subjects. The genomic-inflation factor $(\lambda \mathrm{GC})$ in this analysis was 1.058, indicating that the sample was appropriate for an association study. A quantile-quantile $(\mathrm{Q}-\mathrm{Q})$ plot showed that 2 SNPs deviated from the distribution under the null hypothesis (Additional file 2). Two SNPs on BTA2 reached the Bonferroni-corrected threshold for genome-wide significance $\left(P<1.5 \times 10^{-6}\right.$; Fig. 1a, Table 1$)$. The 2 SNPs, Hapmap43862-BTA-47538 and Hapmap43863-BTA47554, were located within a 200-kbp window from $48,240,577$ bp to $48,440,885$ bp on BTA 2 and were in linkage disequilibrium (LD) with each other $\left(P<6.52 \times 10^{-7}\right.$ $\left.8.32 \times 10^{-7}, D^{\prime}=1, r^{2}=0.99\right)$, as shown in Table 1 . This QTL has not been previously reported for reproductiverelated traits in cattle (reviewed in [14, 15]; for a cattle QTLdb, see [25]).

To characterize the region on BTA2 in more detail-in particular, the extent of LD in the QTL region, the genotypes of 430 animals for 33,303 SNPs were imputed using BEAGLE software [26, 27] with phased haplotype data inferred from 586,812 SNPs (BovineHD Beadchip) in 1,041 Japanese Black cattle served as the reference [23]. We previously estimated the imputation accuracy by comparing the true genotypes to the imputed genotypes, indicating that such imputation was highly accurate [23]. Subsequently, $4 \mathrm{SNPs}$ associated with $\mathrm{ANAI}_{4}$ were detected within the 218-kbp from 48,225,372 bp to $48,443,632$ bp on BTA2 using EMMAX $\left(D^{\prime}=1, r^{2}\right.$ ranging from 0.99 to 1.00; Fig. 1b, Table 1). Except for the 4 SNPs on BTA2, we did not detect any imputed SNPs 
A

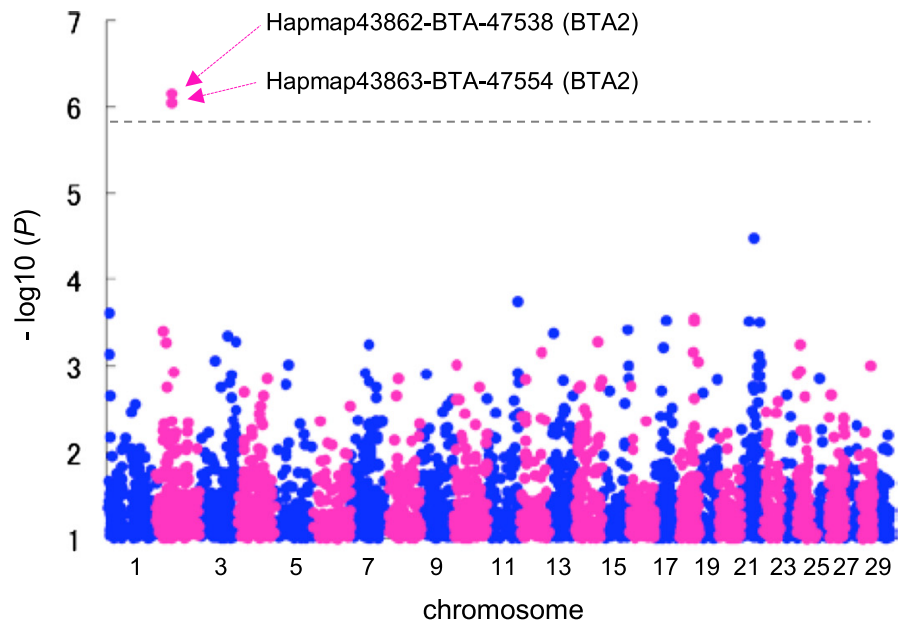

B Hapmap43862-BTA-47538 (50K) Hapmap43863-BTA-47554 (50K) BovineHD0200013961 BovineHD4100001198

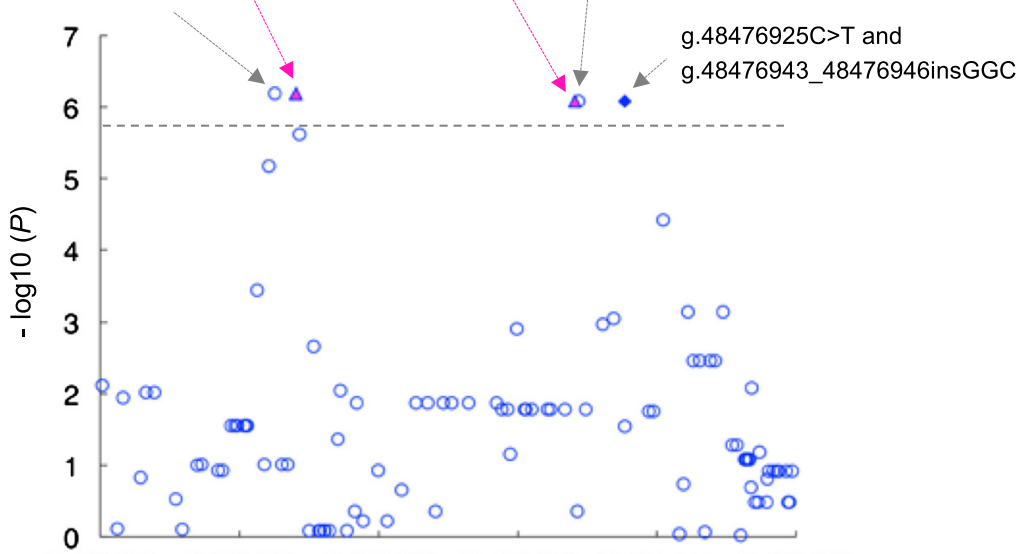

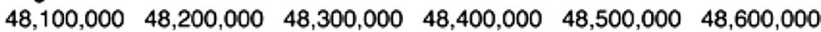

ORC4

C

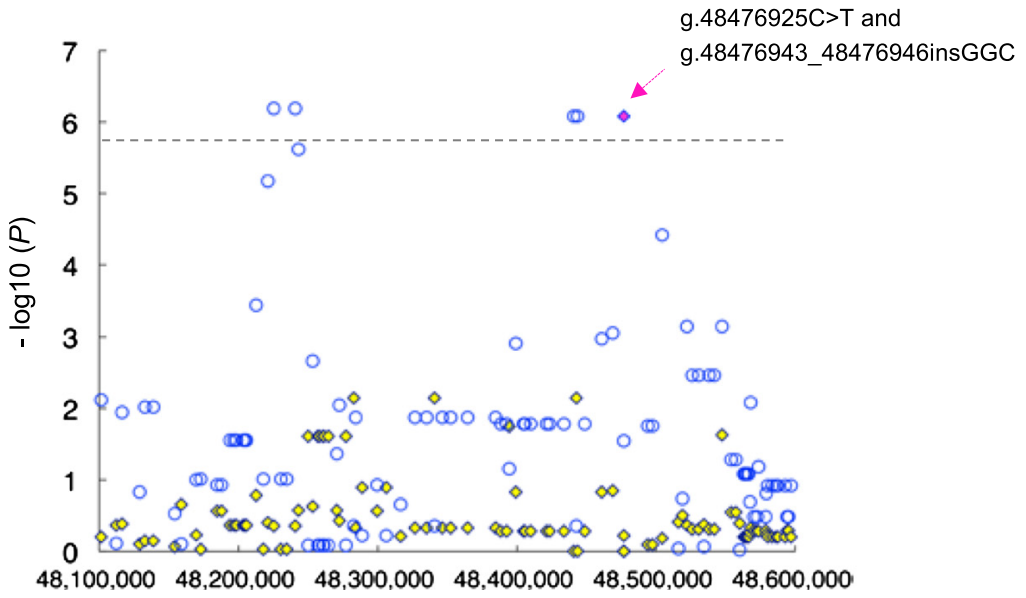

Fig. 1 (See legend on next page.) 
(See figure on previous page.)

Fig. 1 Association of SNPs and a indel with $\mathrm{ANAl}_{4}$ in 430 Japanese Black cattle. a Manhattan plot of the association of 33,303 SNPs (BovineSNP50K BeadChip) with $\mathrm{ANAl}_{4}$ in 430 Japanese Black cattle. The chromosomes are distinguished with alternating colors (blue, odd numbers; red, even numbers). The chromosome number is indicated on the X-axis. The dashed line is the Bonferroni-corrected threshold for genome-wide significance $(-\log 10(P)=5.823)$. The vertical axis is broken for $P$ values below -log10 $(P)=1$. b Regional plot of the locus on BTA2 associated with ANAl 4 . SNPs from the BovineSNP50K BeadChip are shown as red triangles. The imputed SNPs are shown as unfilled blue circles. g.48476925C > T SNP and the g.48476943_48476946insGGC indel are shown as filled blue diamonds. Genes and their directions of transcription are noted at the bottom of the plot. c A conditioned analysis was performed by including the haplotype, defined by g.48476925C > T SNP and the g.48476943_48476946insGGC indel, as covariates in the model. The 2 red, filled diamonds indicate the g.48476925C > T and g.48476943_48476946insGGC (arrow) variants. The blue, unfilled circles and the yellow, filled diamonds represent $P$ values on a -log10 scale before and after conditioning, respectively. The positions shown are based on the UMD3.1 assembly of the bovine genome

that were significantly associated with $\mathrm{ANAI}_{4}(P<0.05$, Bonferroni-corrected).

\section{Variants in the upstream region of $A C V R 2 A$ were associated with $\mathrm{ANAl}_{4}$}

The LD region harbors 2 genes: origin recognition complex subunit 4 (ORC4) and $A C V R 2 A$ (Fig. 1b, Additional file 3). Out of 4 associated SNPs, 2 SNPs were located in the intronic region of $A C V R 2 A$ and 2 SNPs were located on centromeric side at a distance of 42 to $57 \mathrm{kbp}$ from ORC4, respectively (Fig. 1b). To detect associated polymorphisms in ORC4 and $A C V R 2 A$, we sequenced all exons and upstream regions, beginning 2,131 bp upstream of the start codon, of each gene in 3 animals with homozygous $Q$ and $q$ haplotypes that were defined by the genotypes of Hapmap43862-BTA-47538 (48,240,577 bp) and Hapmap43863-BTA-47554 (48,440,885 bp) (Fig. 1a, $\mathrm{B}$, Table 1). In the region of $A C V R 2 A$, we found a 21-bp indel (g.48476691_484766711delGAGCTCGCGGCGGT GGCGGCC) in the $5^{\prime}$-untranslated region (UTR) and a SNP in $3^{\prime}$-UTR (g.48381943A > G) in exons 1 and 11, respectively. We also found a SNP (g.48476925C > T) and a 3-bp indel (g.48476943_48476946insGGC) in the upstream region of $A C V R 2 A$ (716 bp and 737-740 bp upstream of the start codon, respectively). We did not found any variants in the exons or in the region upstream of the ORC4 transcription start site. To ascertain whether the $A C V R 2 A$ variants were associated with $\mathrm{ANAI}_{4}$, we genotyped the variants in 430 animals used in the GWAS and analyzed the association with $\mathrm{ANAI}_{4}$, using EMMAX software and a genetic-relationship matrix for the animals. The SNP (g.48476925C > T) and the 3-bp indel (g.48476943_48476946insGGC) in the upstream region of $A C V R 2 A$ produced a highly significant signal $(P=$ $8.32 \times 10^{-7}$; Fig. 1 b, Table 1$)$, whereas the 21 -bp indel in the $5^{\prime}$-UTR and the SNP in the $3^{\prime}$-UTR of $A C V R 2 A$ were not associated with $\mathrm{ANAI}_{4}(P=0.028$ and $P=$ 0.51 , respectively). Subsequently, 5 SNPs and 1 indel associated with $\mathrm{ANAI}_{4}$ were detected within the 251 . 5 -kbp region spanning 48,225,372 bp to 48,476,946 bp on BTA2 $\left(D^{\prime}=1, r^{2}\right.$ ranging from 0.99 to 1.00 ; Fig. 1b, Table 1).
We then performed conditioned analysis to ascertain whether there were any other significantly associated SNPs in the region. The $Q$ haplotype, defined by the SNP (g.48476925C > T) and the 3-bp indel (g.48476943_48476 946insGGC) in the upstream region of $A C V R 2 A$, was individually included as a covariate in the linear-mixed model. After conditioning, the associations of other SNPs were no longer evident (Fig. 1c), indicating that the region contained a single QTL.

ACVR2A is a type-II transforming growth factor-beta (TGF-beta) receptor with serine/threonine kinase activity, which is involved in initial activin binding. Such binding leads to the recruitment and phosphorylation of type-I TGF-beta receptors and activates transcription of specific target genes, such as the FSH, polypeptide beta gene $(F S H B)$ (reviewed in [28]). FSH is a heterodimer, consisting of an alpha and beta subunit. FSH is produced in gonadotropes in the anterior pituitary gland in response to activin by autocrine and paracrine mechanisms, and stimulates the growth and recruitment of immature follicles in the ovary [29]. Given these facts, $A C V R 2 A$ could be a reasonable candidate gene for the fertility trait.

\section{Variants in the 5'-upstream region of $A C V R 2 A$ were involved in allelic imbalances of ACVR2A mRNA expression}

Variants in the upstream region of the $A C V R 2 A$ gene could potentially affect the activity of the promoter and, thus, they may contribute to an allelic imbalance in ACVR2A mRNA expression. To compare the relative abundance of $Q$ - versus $q$-derived transcripts of $A C V R 2 A$, we performed allelic-imbalance testing with heterozygous samples [30]. The exonic SNPs in 5' and 3 '-UTRs of $A C V R 2 A$ were not associated with $\mathrm{ANAI}_{4}$. The intronic SNPs were transcribed as primary mRNAs in the nucleus; thus, we amplified an intronic $A C V R 2 A$ SNP, BovineHD4100001198 (48,443,632 bp, Table 1), which is in perfect LD with the $Q$ haplotype that was defined by the g.48476925C $>\mathrm{T}$ SNP and the 3bp g.48476943_48476946insGGC indel genotypes $\left(r^{2}=1\right)$. We observed that $A C V R 2 A$ was ubiquitously expressed in female cow tissues including primary dermal fibroblasts 
Table 1 SNPs and a indel with genome-wide significant associations with $\mathrm{ANAl}_{4}$ on BTA2

\begin{tabular}{|c|c|c|c|c|c|c|c|c|c|}
\hline$\overline{\text { BTA }}$ & SNP and indel-ID & Reference SNP-ID_number $^{c}$ & position (bp)_UMD3.1 ${ }^{d}$ & allele_1 $^{\mathrm{e}}$ & $\begin{array}{l}\text { Minor allele frequency } \\
\text { (upper extrame) }\end{array}$ & $\begin{array}{l}\text { Minor allele frequency } \\
\text { (lower extrame) }\end{array}$ & allele_2 ${ }^{f}$ & odds ratio & P-value \\
\hline 2 & BovineHD0200013961 ${ }^{\mathrm{b}}$ & rs110523739 & 48225372 & G & 0.45 & 0.29 & $A$ & 1.98 & $6.52 \mathrm{E}-07$ \\
\hline 2 & Hapmap43862-BTA-47538 & rs41636186 & 48240577 & A & 0.45 & 0.29 & G & 1.98 & $6.52 \mathrm{E}-07$ \\
\hline 2 & Hapmap43863-BTA-47554 & rs41636197 & 48440885 & G & 0.45 & 0.29 & $A$ & 1.96 & $8.32 \mathrm{E}-07$ \\
\hline 2 & BovineHD4100001198 & rs41636199 & 48443632 & A & 0.45 & 0.29 & G & 1.96 & 8.32E-07 \\
\hline 2 & g. $48476925 C>T$ & & 48476925 & $\mathrm{~T}$ & 0.45 & 0.29 & C & 1.96 & $8.32 \mathrm{E}-07$ \\
\hline 2 & g.48476943_48476946insGGC & & 48476943_48476946 & GGC & 0.45 & 0.29 & $(-)$ & 1.96 & $8.32 \mathrm{E}-07$ \\
\hline
\end{tabular}

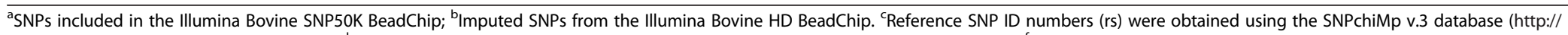

bioinformatics.tecnoparco.org/SNPchimp/). ${ }^{\mathrm{d}}$ The positions are based on the UMD3.1 assembly of the bovine genome. ${ }^{\mathrm{e} M i n o r}$ allele; ${ }^{\mathrm{f}}$ Major allele. The upper and lower extremes correspond to ANAI 4 values above the 85th percentile and below the 15 th percentile, respectively 


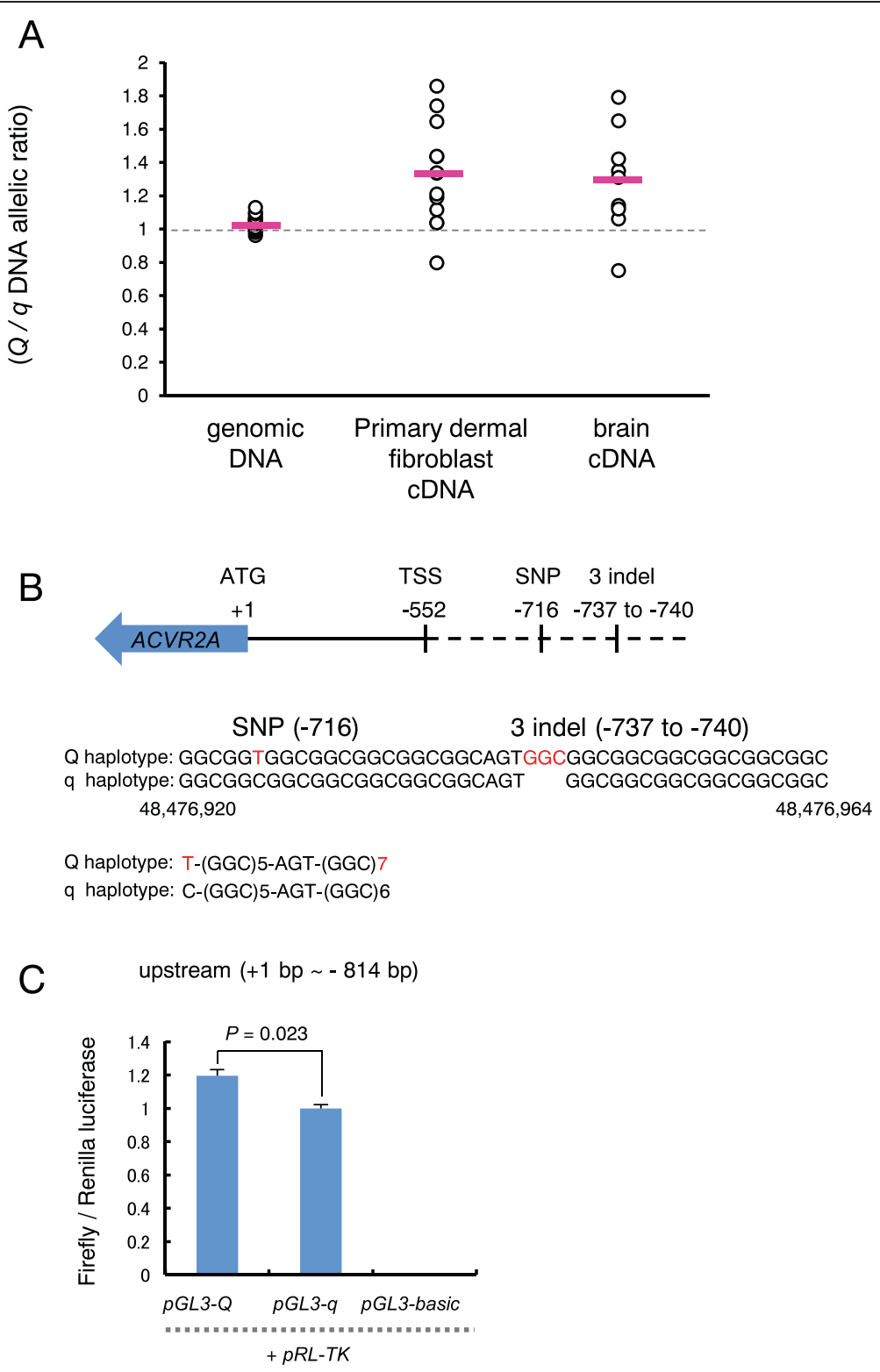

Fig. 2 The upstream region of ACVR2A affects ACVR2A expression. a Allelic-imbalance test for the level of ACVR2A mRNA expression in heterozygotes. CDNA from primary dermal fibroblasts and brain, and gDNA from heterozygous animals were amplified using primers to BovineHD4100001198 (48,443,632 bp), which is located in an intron of ACVR2A (Table 1). The PCR product was directly sequenced. Peak heights at the SNP site were quantified using PeakPicker 2 software [30]. The Y-axis shows the ratio of the peak height of the $Q$ allele to that of the $q$ allele in $g D N A(n=16$, mean $=1.035), c D N A$ from primary dermal fibroblasts $(n=13$, mean $=1.32)$, or cDNA from brains $(n=9$, mean $=1.29)$. Red bars show the mean. The $P$ values for the difference between CDNA and gDNA were 0.009 in primary dermal fibroblasts and 0.044 in brain, respectively, as determined by performing $t$ tests. $\mathbf{b}$ Schematic

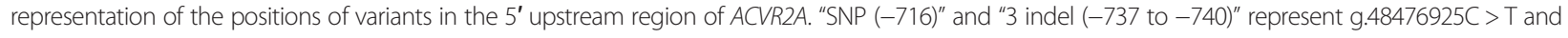
g.48476943_48476946insGGC, respectively. "ATG $(+1)$ " and "TSS (-522)" represent the start codon and the predicted transcriptional start site, respectively. A $Q$ and $q$ haplotype sequence alignment of the upstream region of ACVR2A encompassing 48,476,920 and 48,476,964 bp is shown. c Luciferase reporter assays for the $5^{\prime}$ upstream region of ACVR2A-derived $Q$ and $q$ haplotypes. The $5^{\prime}$ upstream region (814 bp upstream from the start codon) derived from the $Q$ and $q$ haplotypes was cloned into the firefly luciferase pGL3-Q and pGL3- $q$ plasmids, respectively. The Firefly-to-Renilla luminescence ratios observed after cotransfecting HeLa cells were measured to evaluate the effects of the $5^{\prime}$ upstream region. Bars represent the mean \pm SEM obtained in triplicate from 3 independent experiments. $P$ values determined by $t$ tests are shown

and brain tissues (Additional file 4). Therefore, we compared the relative abundances of $Q$ - versus $q$-derived $A C V R 2 A$ transcripts in primary dermal fibroblast $(n=13)$ and brain tissues $(n=9)$ from heterozygotes (Fig. 2a). We isolated samples of genomic DNA (gDNA) and complementary DNA (cDNA) derived from total RNA of whole- 
cell lysates and then compared their allelic ratios using PeakPicker2 software [30]. The results showed that the Qderived ACVR2A transcripts were 1.32-fold and 1.29-fold more abundant than $q$-derived transcripts in primary dermal fibroblasts and in brain ( $t$-test, $P=0.009$ and $P=$ 0.044 , respectively). In contrast, $Q$-derived $A C V R 2 A$ gDNA was detected equally well as $q$-derived gDNA (Fig. 2a).

To determine whether the imbalance in the $A C V R 2 A$ transcript ratio between the haplotypes was attributable to the variants in the upstream region, we cloned the upstream region, beginning 814-bp upstream of the start codon, which included the g.48476925C $>\mathrm{T}$ SNP and the 3-bp indel g.48476943_48476946insGGC from both the $Q$ and $q$ haplotypes, into luciferase-reporter constructs (Fig. 2b). We then transfected HeLa cells with these constructs and measured the resulting luciferase activities at $24 \mathrm{~h}$ post-transfection. The activity was higher for the $Q$ constructs than for the $q$ constructs, with a difference of approximately 1.2 fold ( $t$-test, $P=$ 0.023) (Fig. 2c). These results suggest that the variants including the SNP (g.48476925C > T) and the 3-bp indel (g.48476943_48476946insGGC) in the upstream region of $A C V R 2 A$ affected the level of $A C V R 2 A$ mRNA expression, which could lead to an allelic imbalance in ACVR2A mRNA expression.

We did not observe that the SNP (g.48476925C $>$ T) or the 3-bp indel (g.48476943_48476946insGGC) resided in a transcription factor-binding site in the upstream region of $A C V R 2 A$ in either the $Q$ or $q$ haplotypes, using the TRANSFAC Professional database [31]. The SNP (g.48476925C $>\mathrm{T}$ ) is a T(Q) to $\mathrm{C}(q)$ SNP located 716 bp upstream of the start codon of $A C V R 2 A$, and the 3-bp indel (g.48476943_48476946insGGC) is a $(\mathrm{GGC}) \mathrm{n}$ trinucleotide repeat with either $7(Q)$ or $6(q)$ copies located 737-740 bp upstream of the start codon of $A C V R 2 A$ (Fig. 2b). Recently, Karim et al. reported that 2 causative variants for bovine stature QTL in the upstream region of PLAG1 influence the promoter activity and reflect differential binding of nuclear factors [32]. The causative variants were a (CCG)n trinucleotide repeat with either $11(Q)$ or $9(q)$ copies located immediately upstream of the presumed PLAG1 transcription start site and a $\mathrm{G}(Q)$ to $\mathrm{A}(q)$ SNP located 12-bp upstream of the (CCG)n trinucleotide repeat [32]. The repetitive, GC-rich triplet composition and the location were similar to that observed in the upstream region of $A C V R 2 A$ (Fig. 2b). Therefore, it is possible that the variants in the upstream region of $A C V R 2 A$ could also affect promoter activity and cause differential binding of transcriptional factors between the $Q$ and $q$ haplotype, which in turn may have caused the imbalance observed in $A C V R 2 A$ transcription between animals with $Q$ and $q$ haplotypes.

\section{FSHB expression is dependent on ACVR2A expression in a gonadotrope cell line}

Matzuk et al. [31] found that female Acvr2a-knockout mice were infertile and showed evidence of multiple follicle atresias in the ovaries. Consistently, FSHB expression in the anterior pituitary was suppressed in Acvr2aknockout mice, and serum FSH was decreased in female homozygous Acvr2a-knockout mice $(35.2 \pm 5.2 \mathrm{ng} / \mathrm{ml})$ compared to female wild-type mice $(83.4 \pm 20.6 \mathrm{ng} / \mathrm{ml})$, indicating that ACVR2A affects folliculogenesis by regulating $F S H B$ expression. However, it is unknown whether the level of ACVR2A influences FSHB expression in a dose-dependent manner.

The mouse gonadotrope L $\beta$ T2 cell line $[33,34]$ shows FSHB expression and FSH secretion that is induced in response to activin A through ACVR2A [35-37]. Therefore, this cell type serves as a good model for analyzing whether the level of $A C V R 2 A$ expression influences $F S H B$ expression, using an $F S H B$ promoter-reporter plasmid [36]. Although the transfection efficiency in L $\beta$ T2 cells was very low $(8.8 \pm 1.62 \%$ of 3,368 cells; Additional file 5: Figure S5A) [36], the co-transfection efficiency was approximately $100 \%$ (100 \% of 3,368 cells; Additional file 5: Figure S5B-D). These findings indicated that $F S H B$ promoter-reporter activity was arguably detected in cells co-transfected cells with the ACVR2A expression plasmid. In addition, we confirmed that the level of ACVR2A was dependent on amount of the $A C V R 2 A$ expression plasmid used for the transfections (Additional file 6: Figure S6).

To determine whether the level of $A C V R 2 A$ affects $F S H B$ expression in response to activin A, we cotransfected $\mathrm{L} \beta \mathrm{T} 2$ cells with an $A C V R 2 A$-expression plasmid and an $F S H B$ promoter-reporter plasmid. The results showed that activin A-induced $F S H B$ promoter activity was dependent on the amount of $A C V R 2 A$ expression plasmid used (Fig. 3; Tukey-Kramer post-hoc test, $P<0.01$ ). These finding suggested that the level of $A C V R 2 A$ affected the level of $F S H B$ expression in response to activin A.

Taken together, these results suggested that the variants in the upstream region of $A C V R 2 A$ in the Q-haplotype animals induced an increase in $A C V R 2 A$ transcription relative to that observed in the $q$ animals, which in turn could induce $F S H B$ expression. Cattle are polyestrous animals and display estrous behavior approximately every 21 days. Normally, 2-3 waves of follicular growth occur in the ovaries during each estrous cycle, which are induced by a transient increase of FSH concentration [38-40]. Subsequently, a single dominant follicle is selected in a manner that is dependent on decline of the FSH concentration [41], followed by ovulation of the single dominant follicle or atresia. Although cattle are mono-ovulatory species, FSH concentrations influence the emergence of co- 


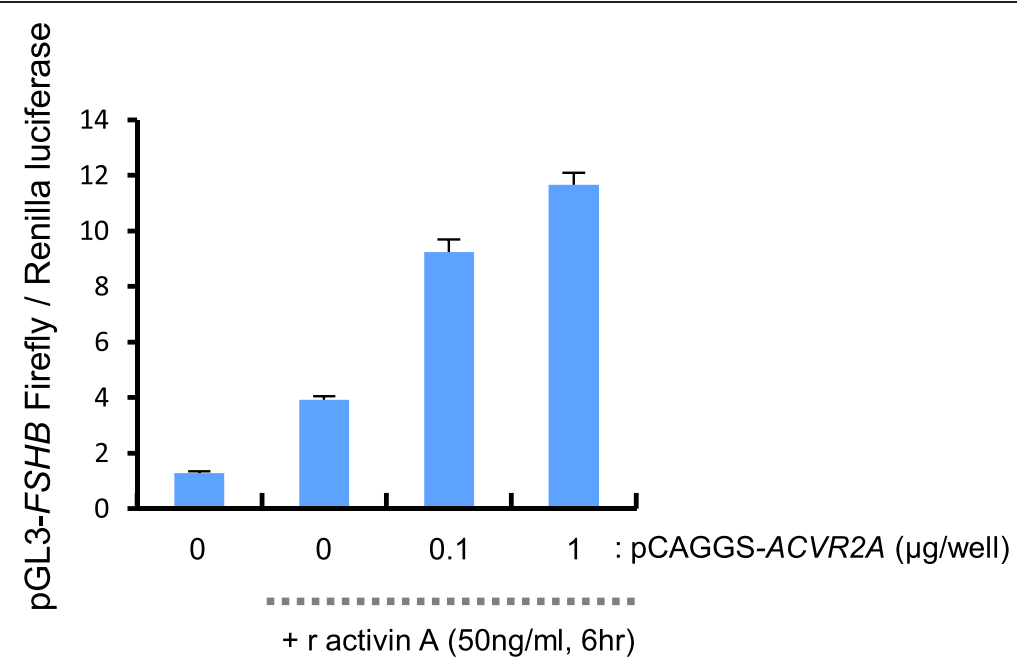

Fig. 3 FSHB promoter activity is dependent on ACVR2A expression in response to activin A in LBT2 cells. The pGL3-Basic-FSHB (chr2:107,059, 651-107,061,641 on the GRCm38/mm10 mouse genome assembly) and PCAGGS-ACVR2A plasmids were cotransfected with pRL-TK into L $\beta$ T2 cells. At $18 \mathrm{~h}$ post-transfection, recombinant activin A was added for $6 \mathrm{~h}$, and the Firefly-to-Renilla luminescence ratios were measured. Bars represent the mean luminescence \pm SEM obtained in triplicate from 3 independent experiments. $P$ values were determined by Tukey-Kramer post-hoc test $(P<0.01)$

dominant follicles [42]. Moreover, administration of exogenous recombinant $\mathrm{FSH}$ in cattle induces multiple follicles growth during super-ovulation [43]. In this manner, follicular growth at several stages is closely associated with circulating FSH concentrations. Therefore, although quantitative differences in FSHB expression between $Q$ and $q$-haplotype animals may be subtle, it is possible that these differences govern folliculogenesis. Thus, further analyses regarding differences of serum FSH levels and follicle size at each stage between $Q$ and $q$-haplotype animals could help to elucidate the mechanisms underlying the variants of $A C V R 2 A$ that influence female fertility.

Of note, mutation of TGF-beta family ligand and its receptor BMP15 [44] and the type-I receptor ALK6 [45, 46] were identified as causative variants in the ovulation rate in sheep. Inactivation of 1 copy of BMP15 increased the ovulation rate, whereas inactivation of both copies of BMP15 showed blocked follicular growth. In contrast, ALK6 mutant alleles increased the ovulation rate in an additive manner, indicating that the TGF-beta family ligand and its receptor functions in folliculogenesis in a dosage-sensitive manner. Similarly, ACVR2A, which is a TGF-beta family type II receptor, may also function in female fertility in cattle in a dose-dependent manner.

\section{Replication of the associated haplotype in a sample of 1,433 animals}

To validate the GWAS results and estimate the effective size of the QTL and the allele frequencies, we examined whether the $Q$ haplotype was associated with $\mathrm{ANAI}_{4}$ in a sample of 1,433 animals. We genotyped 1,433 animals randomly selected from the remainder of the cohort from the same farm used for the GWAS (Additional file 7 ). The results showed that the $Q$ haplotype was significantly associated with $\mathrm{ANAI}_{4}$ compared to the $q$ haplotype (Tukey-Kramer post-hoc test, $P=0.017$; Table 2). The $Q$ haplotype frequency was 0.39 , indicating that the haplotype is common in Japanese Black cattle. We fitted a linear-mixed model to the $\mathrm{ANAI}_{4}$ values in the additive model and used restricted maximum likelihood (REML) analysis to estimate the variance explained by the haplotype. We estimated the proportion of total genetic variance attributable to the $Q$ haplotype as 0.1 (Table 2). The Q-to- $q$ haplotype substitution effect on $\mathrm{ANAI}_{4}$ was 0.02 (Table 2).

\section{Conclusion}

This GWAS identified variants in the upstream region of $A C V R 2 A$, which were associated with female fertility in Japanese Black cattle. The variants affected the level of $A C V R 2 A$ mRNA expression, which led to an allelic imbalance. Expression of exogenous $A C V R 2 A$ induced dose-dependent increases of FSHB expression in response to activin A. Finally, we replicated this association and estimated the effect in a sample of 1,433 animals. Thus, the results suggest that $Q$ haplotype could serve as a useful marker in Japanese Black cattle to select animals with superior female fertility in Japanese Black cattle.

\section{Methods}

\section{Ethics statement}

All animal experiments were performed according to the guidelines for care and use of laboratory animals of 
Table 2 Proportion of genetic variance attributable to haplotypes associated with $\mathrm{ANAl}_{4}$

\begin{tabular}{|c|c|c|c|c|c|c|c|c|c|}
\hline BTA & $\begin{array}{l}\text { BTA SNP } \\
\text { and indel-id }\end{array}$ & positon (bp) _UMD3,1 ${ }^{\text {a }}$ & Q/a haplotype & $\begin{array}{l}\text { Number of } \\
\text { animals } \\
\text { genotyped } \\
\text { for the haplotype }\end{array}$ & $\begin{array}{l}\text { Minor allele } \\
\text { frequency }\end{array}$ & Heritability & $\begin{array}{l}\text { Haplotype } \\
\text { effect on } \\
\text { total genetic } \\
\text { variance }^{b}\end{array}$ & $\begin{array}{l}\text { Haplotype } \\
\text { substitution } \\
\text { P-value }^{d} \\
\text { effect }^{c}\end{array}$ & P-value ${ }^{d}$ \\
\hline 2 & $\begin{array}{l}\text { g. } 48476925 C>T \text { and } \\
\text { g.48476943_48476946insGGC }\end{array}$ & $\begin{array}{l}48476925 \text { and } \\
48476943.48476946\end{array}$ & $\begin{array}{l}\text { T and GCC/ } \\
C \text { and }(-)\end{array}$ & 1433 & 0.39 & 0.02 & 0.1 & 0.02 & 0.017 \\
\hline
\end{tabular}

\section{aThe positions shown are based on the UMD3.1 assembly of the bovine genome.}

${ }^{\mathrm{b}}$ The effects of the haplotype were estimated as the least-square means from GLM analysis. The statistical model for GLM analysis included the fixed variables of the farm, birth year, and haplotype. The genetic

variance explained by the haplotype was calculated based on estimates of the haplotype effect and the haplotype frequency [55]. Total genetic variance was estimated using the MTDF-REML programs. The effect size of haplotype was estimated as the proportion of genetic variance explained by the haplotype.

${ }^{c}$ The average $\mathrm{ANAl}_{4}$ values for $\mathrm{QQ}$ and $q q$ were 0.69 and 0.65 , respectively.

dResults were tested by a 1-way ANOVA, followed by the Tukey-Kramer test for multiple comparisons 
Shirakawa Institute of Animal Genetics, and this research project was approved by the Shirakawa Institute of Animal Genetics Committee on Animal Research (H21-1). We have obtained the written agreement from the cattle owners to use the samples and data.

\section{Collection of phenotype data}

Data were collected from cattle farms, and the data management system for Japanese Black cattle was described in a previous study $[47,48]$. The original data included 63,775 records for reproductive females born from 1992 to 2006. The data were selected using 9 selection criteria: 1) data were not missing for the cow from first to fourth parity, 2) the cow did not have twins in parturition, 3) the cow did not receive any embryo transfers, 4) the cow did not have any abortions, 5) the length of all gestations ranged from 261 to 310 days, 6) the calving interval ranged from 276 to 730 days, 7) the age of the cow at the first calving was be less than 1,128 days, 8) the cow was reared in a single farm, and 9) each breeding farm had more than 10 records from each birth year. After applying these selection criteria, the final dataset contained 10,399 records. In this study, female fertility was evaluated as a metric to describe the average inverse of the number of artificial inseminations required for conception [20] from the first parity through the fourth parity. For example, if conception is achieved at the first, second, third, or fourth AI, ANAI values are $1,0.5,0.33$, and 0.25 , respectively. $\mathrm{ANAI}_{4}$ values were corrected for the effects of the individual farm and birth year. The heritability and variance components of phenotypic variance were estimated with the REML procedure using the MTDF-REML programs [49]. We fitted single-traits animal models with random effects and fixed effects:

$$
y_{i j}=\mu+\text { year }_{i}+\text { farm }_{j}+u_{i j}+e_{i j}
$$

where $y_{i j}$ is the observation of $i j$ for the traits, $\mu$ is the total mean, year $r_{i}$ is the fixed effect of birth year $i$ (15 classes, 1992 to 2006), farm $_{j}$ is the fixed effect of farm $j$ (174 classes), and $u_{i j}$ is the infinitesimal genetic effect of animal $i j$, which is distributed as $N\left(0, \mathbf{A} \sigma_{u}^{2}\right)$, where $\mathbf{A}$ is the numerator relationship matrix, and $e_{i j}$ is the residual effect. Pedigrees of the base population animals were traced back for 2 generations to create the numerator relationship matrix, and 10,399 animals were included in the pedigree analysis.

\section{Selection of samples for the GWAS and DNA sample collection}

Samples were selected from the upper extreme $\left(85^{\text {th }}\right.$ percentile, 1,560 animals $)$ and the lower extreme $\left(15^{\text {th }}\right.$ percentile, 1,560 animals) for $\mathrm{ANAI}_{4}$. To reduce population stratification, we selected less than 5 cows derived from a single sire in each extreme, resulting in 256 cows for the upper extreme and 174 cows for the lower extreme. Whole blood was collected from each cow, and gDNA was isolated using the Easy-DNA gDNA Purification Kit (Invitrogen, Cat. \#K1800-01).

\section{GWAS for ANAI}

A total of 430 DNA samples were genotyped using the BovineSNP50K BeadChip (version 1, Illumina), which comprises probes for 54,001 SNPs. The UMD3.1 assembly [50] was used to map the positions of the SNPs. The data were analyzed using PLINK software, v1.07 [51]. A total of 33,303 autosomal SNPs that passed our quality control criteria (call rate $>99 \%$, minor allele frequency $>0.01$, Hardy-Weinberg equilibrium $P>$ 0.001 , and inclusion of the SNP on the Illumina Bovine HD BeadChip) [23] were used for the association study. We performed a GWAS using the trait as a binary variable, as is commonly done in case-control studies. To analyze the upper- and lower-performance phenotypes, we used a linear mixed model with a genetic relationship matrix for the binary phenotypes using the EMMAX software [24].

\section{Linkage disequilibrium and diplotype analysis}

Haploview 4.2 software [52] was used to analyze linkage disequilibria between the SNPs. The diplotypes of the GWAS samples were estimated using BEAGLE3.3.2 software $[26,27]$.

\section{Imputation of SNPs}

The genotypes of 33,303 SNPs were imputed using BEAGLE 3.3.2 software, with phased haplotype data inferred from 586,812 SNPs in 1,041 Japanese Black cattle as the reference [23].

\section{Expression analysis}

For real-time quantitative PCR, we extracted total RNA from cow tissues, primary bovine dermal fibroblasts, and bovine endometrial epithelial cells (Cell Application, Inc., Cat. \#B932-05) using RNeasy Mini Kits (Qiagen, Cat. \#74104), after which the total RNA was treated with DNase I. cDNA was synthesized from 50 ng total RNA using the ReverTra Ace- $\alpha$ Kit (Toyoba, Cat. \#FSK-101) with random primers, according to the manufacturer's instructions. The $A C V R 2 A$ gene was detected with the following primers and probe: forward, $55^{\prime}$-catgggatt agtcctgtgggaac- $3^{\prime}$; reverse, $5^{\prime}$-cctcaaatggcagcatgtattca-3'; and probe, 5'-tacaggtccatctgcagcagtacagcga-3'. Realtime PCR was performed on a 7900HT Real-Time PCR System (Applied Biosystems) using the comparative Ct method with glyceraldehyde-3-phosphate dehydrogenase mRNA serving as the internal control. 


\section{Allelic imbalance test}

To quantify the allelic imbalance of $A C V R 2 A$ transcripts, we designed PCR primers to BovineHD4100001198 $(48,443,632 \mathrm{bp})$ on BTA2, located in an intron of $A C V R 2 A$. The forward primer was $5^{\prime}$-aacctagaaaccgtag aaagacga-3', and the reverse primer was $5^{\prime}$-gatggc atctcttggctcat-3'. We used 50 ng of template cDNA from primary bovine dermal fibroblasts or bovine brain tissues (medulla oblongata), or $10 \mathrm{ng}$ of gDNA from heterozygous animals for PCR amplification with TaKaRa Ex Taq HS DNA Polymerase (TaKaRa, Cat. \#RR006). The PCR product was directly sequenced and purified with the CleanSEQ system (Agencourt, Cat. \#A29154). Peak heights at polymorphic sites were quantified using PeakPicker 2 software [30]. Allelic imbalances were estimated as the ratio of the peak height of the $Q$ allele to that of the $q$ allele in cDNA and in gDNA. Calibration curves were generated using data obtained by mixing varying amounts of gDNA from $Q$ and $q$ homozygotes.

\section{Luciferase reporter assays}

To measure the effects of the 5 '-upstream region of $A C V R 2 A$ on $A C V R 2 A$ expression, the 814-bp fragment upstream of the start codon of $A C V R 2 A$ of each haplotype ( $Q$ and $q$ haplotype) including a SNP (g.48476925C > T) and a 3-bp indel (g.48476943_48476946insGGC), was PCR amplified using PrimeSTAR Max DNA Polymerase (Takara, Cat. \#R045A). PCR was performed using gDNA, a forward primer (5'-GGGGTACCacaatctcctcgcgctcac-3'; uppercase letters indicate the $K p n I$ linker), and a reverse primer (5' -TCCCCCGGGactttgcagcagctcccatt-3'; uppercase letters indicate the SmaI linker). The PCR products were cloned into the KpnI and SmaI sites of the pGL3Basic Vector (Promega, Cat. \#E1751). The sequence and orientation of the insert were confirmed by sequencing. The pGL3-Basic Vector was used for mock transfections. For cell culture, HeLa S3 cells were maintained in Dulbecco's modified Eagle's medium (DMEM; Sigma, Cat. \#D5796) with $10 \%$ fetal calf serum (FCS; Sigma, Cat. \#F2442) supplemented with $2 \mathrm{mM}$ L-glutamine (Gibco, Cat. \#25030-081) and 100 units $/ \mathrm{ml}$ penicillin and $100 \mu \mathrm{g} / \mathrm{ml}$ streptomycin (Gibco, Cat. \#15140-122). Using Lipofectamine 2000 (Invitrogen, Cat. \#11668-019), we transfected $5 \times 10^{4}$ cells per well in a 24-well plate with a mixture of $750 \mathrm{ng}$ of the reporter vector and $10 \mathrm{ng}$ of the pRL-TK Renilla vector (Promega, Cat. \#E2241) to calibrate transfection efficiency. Luciferase assays were performed $24 \mathrm{~h}$ post-transfection using the Dual Luciferase Reporter Assay System (Promega, Cat. \#E1910) and the GloMax (Promega, Cat. \#E6521).

\section{FSHB promoter-reporter assay}

To measure the $F S H B$ promoter activity, the promoter region of FSHB (chr2:107,059,651-107,061,641 on the
GRCm38/mm10 mouse genome assembly) [36] was PCR amplified from gDNA of a C57BL/6NJ mouse using a forward primer (5'-GGGGTACCcctgttcattaaccactgagct-3'; uppercase letters indicate the KpnI linker) and a reverse primer (5'-CCGCTCGAGcactgagtcaagttacacctca-3'; uppercase letters indicate the $X h o \mathrm{I}$ linker). The PCR products were cloned into the KpnI and XhoI sites of pGL3Basic. The sequence and orientation of the insert were confirmed by sequencing. To express the ACVR2A protein, the coding region of ACVR2A (NM_007396, 6642,205 bp) was PCR amplified from cDNA from L $\beta$ T2 cells using a forward primer (5'-GCTCTAGAatgggagctgctgC aaagttggc- $3^{\prime}$; uppercase letters indicate the XhaI linker) and a reverse primer (5'-CGGAATTCctaagcgtaatcagg aacgtcgtaagggtatagactagattctttgggaggaaagtc- 3 '; uppercase letters indicate the EcoRI linker, and underlined letters indicate the C-terminal hemagglutinin [HA] tag for ACVR2A, respectively). The PCR product was cloned into the XhaI and EcoRI sites of the pCAGGS vector [53]. The sequence and orientation of the insert were confirmed by sequencing. The expression of ACVR2A was confirmed by western blotting with an anti-HA antibody 3 F10 (Roche, Cat. \#11 867423 001, 100 ng/ml). Immunoreactivity was detected with a horseradish peroxidaseconjugated donkey anti-rat IgG antibody (Jackson ImmunoResearch, Cat. \#712-035-153) and the ECL Prime Western Blotting Detection Reagent (GE Healthcare, Cat. \#RPN2232). Chemiluminescence was detected with an ImageQuant LAS 4000 (GE Healthcare) and quantified using the ImageQuant TL Analysis Toolbox. L $\beta$ T2 cells $[33,34]$ were maintained in DMEM with $10 \%$ charcoal-stripped FCS (Gibco, Cat. \#12676-029) supplemented with $2 \mathrm{mM}$ L-glutamine, $20 \mathrm{nM}$ dexamethasone (Sigma, Cat. \#D4902), $0.1 \mathrm{mM}$ non-essential amino acids (Gibco, Cat. \#11140-050), and 100 units/ml penicillin and $100 \mu \mathrm{g} / \mathrm{ml}$ streptomycin.

To determine whether the expression level of $A C V R 2 A$ affected $F S H B$ reporter activity in response to activin A, we transfected $2 \times 10^{5}$ cells per well in a 24 -well plate with a mixture of $200 \mathrm{ng}$ of the $F S H B$ promoter-reporter vector, pCAGGS-ACVR2A (using the amounts of plasmid indicated in Fig. 3) and $10 \mathrm{ng}$ of pRL-TK Renilla to determine transfection efficiencies. After $18 \mathrm{~h}, 50 \mathrm{ng} / \mathrm{ml}$ recombinant activin A (R\&D, Cat. \#338-AC; $50 \mu \mathrm{g} / \mathrm{ml}$ stock in $0.1 \%$ bovine serum albumin/phosphate-buffered saline) was added to the culture medium, and luciferase assays were performed at $6 \mathrm{~h}$ post-activin A stimulation using the Dual Luciferase Reporter Assay System and the GloMax.

To examine co-transfection efficiencies in L $\beta$ T2 cells, we used the pCAGGS-EGFP (Clontech, Cat. \#6085-1) and pCAGGS-mCherry (Clontech, Cat. \#632522) vectors. At $24 \mathrm{~h}$ post-transfection, cells were examined with a confocal microscope (FV1000, Olympus Optical) and 
fluorescence-positive cells were counted using ImageJ software, version 1.46 [54].

\section{Replication study}

For the replication study, we used 1,433 samples from the remainder of the cohort from the same farm selected for the GWAS (Additional file 7). The g.48476925C $>$ T SNP was genotyped by directly sequencing PCR products using a forward primer ( $5^{\prime}$-acaatctcctcgcgctcac- 3 ') and a reverse primer ( $5^{\prime}$-caagttctggtccaggctct- $\left.3^{\prime}\right)$. PCR products were sequenced using the reverse primer and the BigDye Terminator v.3.1 Cycle Sequencing Kit (Applied Biosystems), followed by electrophoresis using an ABI 3730 sequencer (Applied Biosystems) and genotyping using SeqScape software, V2.5 (Applied Biosystems). The 3-bp indel (g.48476943_48476946insGGC) was PCR-amplified using a forward primer $\left(5^{\prime}\right.$-acaatctcctcg cgctcac- $\left.3^{\prime}\right)$ and a reverse primer ( $5^{\prime}$-fluorescein amiditecaagttctggtccaggctct- $\left.3^{\prime}\right)$. PCR products were electrophoresed using an ABI 3730 sequencer (Applied Biosystems) and genotyped using GeneScan analysis software (Applied Biosystems) and GeneMapper software v3.7 (Applied Biosystems).

\section{Estimation of the genetic variance explained by the haplotype and effect size of haplotype}

The effects of the haplotype were estimated as the least square means in generalized linear model (GLM) analyses. The statistical model for GLM analysis included fixed variables, such as the farm, birth year, and haplotype. The genetic variance explained by the haplotype was calculated based on estimates of the haplotype effect and the haplotype frequency [55]. Total genetic variance was estimated using the MTDF-REML program. The effect size of haplotype was estimated as the proportion of genetic variance explained by the haplotype.

\section{Availability of supporting data}

The data sets supporting the results of this article are included within the article and its additional file.

\section{Additional files}

\section{Additional file 1: Distribution of $\mathrm{ANAl}_{4}$ in 10,399 cows in this study. (PDF $31 \mathrm{~kb}$ ) \\ Additional file 2: Quantile-quantile plots of the GWAS results for $\mathbf{A N A l}_{4}$. The red dots represent the observed $-\log 10 P$ values, and the straight line represents the expected $-\log 10 P$ values under the null hypothesis. (PPTX $68 \mathrm{~kb}$ )}

Additional file 3: Genes on $\mathrm{BTA2}$ in the $\mathrm{ANAl}_{4}$-associated region. The positions are based on the UMD3.1 assembly of the bovine genome. (XLSX $36 \mathrm{~kb}$ )

Additional file 4: Relative expression of ACVR2 in cow tissues and cells. Relative ACVR2A expression levels in tissues and cells are indicated on the Y-axis. Total RNA was extracted from tissues (1-16), primary dermal fibroblasts (17) from 2 female Japanese Black cattle, or from bovine primary endometrial epithelial cells (18). Relative gene expression levels in the different tissues are shown as mean quantities relative to the value observed in ovarian tissue (dotted line). (PPTX $50 \mathrm{~kb}$ )

Additional file 5: Transfection and co-transfection efficiencies of $L \beta T 2$ cells. (A) To examine the transfection efficiency of $L \beta T 2$ cells, we used the pCAGGS-EGFP and pCAGGS-mCherry vectors. At $24 \mathrm{~h}$ post-transfection, double fluorescence-positive cells were counted using ImageJ software. The data shown represent the transfection efficiencies of $L \beta T 2$ cells in 5 experiments. The average transfection efficiency was $8.8 \pm 1.62 \%$ (3,368 cells). (B-D) To examine co-transfection efficiency in $\llcorner\beta T 2$ cells, we used the pCAGGS-EGFP and pCAGGS-mCherry vectors. At $24 \mathrm{~h}$ post-transfection, double fluorescence-positive cells (B, magenta) were counted using ImageJ software. All GFP positive cells ( $C$, green) were mCherry-positive $(D, r e d)$, representing $100 \%$ of 3,368 cells. (PPTX $6646 \mathrm{~kb}$ )

Additional file 6: Expression of pCAGGS-ACVR2A in L $\beta T 2$ cells. (A) To determine whether the PCAGGS-ACVR2A plasmid was expressed in L $\beta T 2$ cells, we transfected $2 \times 10^{5}$ cells per well in a 24-well plate with a mixture of $200 \mathrm{ng}$ of the FSHB promoter-reporter plasmid, pCAGGSACVR2A (the amount of each plasmid is indicated in the lanes) and $10 \mathrm{ng}$ of pRL-TK Renilla. HA-ACVR2A expression was confirmed by western blot analysis using an anti-HA antibody. Unstained Precision Plus protein standards were used as a marker (BioRad, Cat. \#161-0363). HA-ACVR2A expression was detected with multiple bands ( $57.8 \mathrm{kDa})$ because TGFbeta type-Il receptors are normally modified by phosphorylation and glycosylation, causing then to migrate heterogeneously in sodium dodecyl sulfate-polyacrylamide gel electrophoresis gels. (B) Relative ACVR2A band intensities were measured using the ImageQuant TL Analysis Toolbox. The amounts of loaded proteins were calibrated by Coomassie Brilliant Blue-stained bands between 37 and $75 \mathrm{kDa}$. The bars represent the mean \pm SEM observed in triplicate from 3 independent experiments. (PPTX $706 \mathrm{~kb}$ )

Additional file 7: Distribution of $\mathrm{ANAl}_{4}$ in 1,433 cows for the replication study. A sample population $(n=1,433)$ was derived from the remainder of the cohort from the same farm used for the GWAS. (PPTX $47 \mathrm{~kb})$

\section{Abbreviations}

$\mathrm{ANAl}_{4}$ : Average of the inverse of the number of artificially inseminations required for conception from first through fourth parity; BTA: Bovine (Bos taurus) chromosome; GWAS: Genome-wide association study; LD: Linkage disequilibrium; PCR: Polymerase chain reaction; QTL: Quantitative trait locus or loci; SNP: Single nucleotide polymorphism.

\section{Competing interests}

The authors declare no conflict of interest.

\section{Authors' contributions}

SS and TI conceived and designed the study. SS, YS performed SNP genotyping. SS performed the GWAS analysis. TI collected and analyzed the data. SS and TI performed replication studies. SS, TM, KT, SI, and MS performed the expression and functional analyses. SS, TI, and YS wrote the manuscript. All authors read and approved the final manuscript.

\section{Acknowledgements}

We would like to thank Toshio Watanabe helping with the data analysis, as well as Takatoshi Kojima and other lab members for providing generous support and valuable suggestions. We are grateful to Pamela Mellon for the L $\beta$ T2 cells and Jun-ichi Miyazaki for the pCAGGS plasmid. We are grateful to Emiko Watanabe for technical assistance. This work was supported by funding from the KIEIKAI Research Foundation (H24-KIEIKAI and H25-KIEIKAI) to SS, the JSPS KAKENHI (Grant Number 26450384) to SS, and the Japan Racing and Livestock Promotion to YS.

\section{Author details}

${ }^{1}$ National Livestock Breeding Center, Odakura, Nishigo, Fukushima 961-8511, Japan. ${ }^{2}$ Graduate School of Environmental and Life Science, Okayama University, Tsushima-naka, Okayama 700-8530, Japan. ${ }^{3}$ Gifu Prefectural 
Livestock Research Institute, Kiyomi, Takayama, Gifu 506-0101, Japan. ${ }^{4}$ Cattle Breeding Development Institute of Kagoshima Prefecture, Osumi, So, Kagoshima 899-8212, Japan. ${ }^{5}$ Shirakawa Institute of Animal Genetics, Japan Livestock Technology Association, Odakura, Nishigo, Fukushima 961-8061, Japan.

Received: 11 July 2015 Accepted: 16 October 2015 Published online: 20 October 2015

\section{References}

1. Annual report of conceptional rate in Japan, Livestock Improvment Association of Japan. [http://liaj.or.jp/giken/gijutsubu/seieki/jyutai.htm]

2. Lucy MC. Reproductive loss in high-producing dairy cattle: where will it end? J Dairy Sci. 2001;84(6):1277-93.

3. Matukumalli LK, Lawley CT, Schnabel RD, Taylor JF, Allan MF, Heaton MP, et al. Development and characterization of a high density SNP genotyping assay for cattle. PLoS One. 2009;4(4):e5350.

4. Rincon G, Weber KL, Eenennaam AL, Golden BL, Medrano JF. Hot topic: performance of bovine high-density genotyping platforms in Holsteins and Jerseys. J Dairy Sci. 2011;94(12):6116-21.

5. Kim ES, Berger PJ, Kirkpatrick BW. Genome-wide scan for bovine twinning rate QTL using linkage disequilibrium. Anim Genet. 2009;40(3):300-7.

6. Huang W, Kirkpatrick BW, Rosa GJ, Khatib H. A genome-wide association study using selective DNA pooling identifies candidate markers for fertility in Holstein cattle. Anim Genet. 2010;41(6):570-8.

7. Sahana G, Guldbrandtsen B, Bendixen C, Lund MS. Genome-wide association mapping for female fertility traits in Danish and Swedish Holstein cattle. Anim Genet. 2010;41(6):579-88.

8. Olsen HG, Hayes BJ, Kent MP, Nome T, Svendsen M, Lien S. A genome wide association study for QTL affecting direct and maternal effects of stillbirth and dystocia in cattle. Anim Genet. 2010;41(3):273-80.

9. Olsen HG, Hayes BJ, Kent MP, Nome T, Svendsen M, Larsgard AG, et al. Genome-wide association mapping in Norwegian Red cattle identifies quantitative trait loci for fertility and milk production on BTA12. Anim Genet. 2011;42(5):466-74.

10. Sahana G, Guldbrandtsen B, Lund MS. Genome-wide association study for calving traits in Danish and Swedish Holstein cattle. J Dairy Sci. 2011;94(1):479-86.

11. Schulman NF, Sahana G, Iso-Touru T, McKay SD, Schnabel RD, Lund MS, et al. Mapping of fertility traits in Finnish Ayrshire by genome-wide association analysis. Anim Genet. 2011;42(3):263-9.

12. Hawken RJ, Zhang YD, Fortes MR, Collis E, Barris WC, Corbet NJ, et al. Genome-wide association studies of female reproduction in tropically adapted beef cattle. J Anim Sci. 2012;90(5):1398-410.

13. Sugimoto M, Sasaki S, Gotoh Y, Nakamura Y, Aoyagi Y, Kawahara T, et al. Genetic Variants Related to Gap Junctions and Hormone Secretion Influence Conception Rates in Cows. Proc Natl Acad Sci U S A. 2013;110(48):19495-500.

14. Fortes MR, Deatley KL, Lehnert SA, Burns BM, Reverter A, Hawken RJ, et al. Genomic regions associated with fertility traits in male and female cattle: advances from microsatellites to high-density chips and beyond. Anim Reprod Sci. 2013;141(1-2):1-19.

15. Kirkpatrick BW. Genetics of Reproduction in Cattle. In: Garrick DJ, Ruvinsky A, editors. The Genetics of Cattle. 2nd ed. Boston: CABI Publishing; 2014: 260-283

16. Cameron PJ, Zembayashi M, Lunt DK, Mitsuhashi T, Mitsumoto M, Ozawa S, et al. Relationship between Japanese beef marbling standard and intramuscular lipid in the M. longissimus thoracis of Japanese Black and American Wagyu Cattle. Meat Sci. 1994;38(2):361-4.

17. Namikawa K. Japanese Beef Cattle - Historical Breeding Processes of Japanese Beef Cattle and Preservation of Genetic Resources as Economic Farm Animal (in Japanese): Wagyu Registry Association. Wagyu. 1992.

18. McKay SD, Schnabel RD, Murdoch BM, Matukumalli LK, Aerts J, Coppieters W, et al. An assessment of population structure in eight breeds of cattle using a whole genome SNP panel. BMC Genet. 2008;9:37.

19. Wagyu Registry Association. The breeding value estimation for the number of calves produced at a specific age (in Japanese). Wagyu. 2010;252(61):40-8.

20. Weller Jl, Ezra E. Genetic analysis of somatic cell score and female fertility of Israeli Holsteins with an individual animal model. J Dairy Sci. 1997;80(3):586-93.
21. Kirkpatrick BW. Genetics and Biology of Reproduction in Cattle. In: Fries R, Ruvinsky A, editors. The Genetics of Cattle. Boston: CABI Publishing; 1999: 391-410.

22. Xing C, Xing G. Power of selective genotyping in genome-wide association studies of quantitative traits. BMC Proc. 2009;3 Suppl 7:S23.

23. Sasaki S, Ibi T, Watanabe T, Matsuhashi T, Ikeda S, Sugimoto Y. Variants in the 3' UTR of General Transcription Factor IIF, polypeptide 2 affect female calving efficiency in Japanese Black cattle. BMC Genet. 2013;14:41.

24. Kang HM, Sul JH, Service SK, Zaitlen NA, Kong SY, Freimer NB, et al. Variance component model to account for sample structure in genome-wide association studies. Nat Genet. 2010;42(4):348-54.

25. CattleQTLdb. [http://www.animalgenome.org/cgi-bin/QTLdb/BT/index]

26. Browning SR, Browning BL. Rapid and accurate haplotype phasing and missing-data inference for whole-genome association studies by use of localized haplotype clustering. Am J Hum Genet. 2007;81(5):1084-97.

27. Browning BL, Browning SR. A unified approach to genotype imputation and haplotype-phase inference for large data sets of trios and unrelated individuals. Am J Hum Genet. 2009:84(2):210-23.

28. Bernard DJ, Tran S. Mechanisms of activin-stimulated FSH synthesis: the story of a pig and a FOX. Biol Reprod. 2013;88(3):78.

29. Kumar TR, Wang Y, Lu N, Matzuk MM. Follicle stimulating hormone is required for ovarian follicle maturation but not male fertility. Nat Genet. 1997;15(2):201-4.

30. Ge B, Gurd S, Gaudin T, Dore C, Lepage P, Harmsen E, et al. Survey of allelic expression using EST mining. Genome Res. 2005;15(11):1584-91.

31. Matys V, Kel-Margoulis OV, Fricke E, Liebich I, Land S, Barre-Dirrie A, et al. TRANSFAC and its module TRANSCompel: transcriptional gene regulation in eukaryotes. Nucleic Acids Res. 2006;34(Database issue):D108-110.

32. Karim L, Takeda H, Lin L, Druet T, Arias JA, Baurain D, et al. Variants modulating the expression of a chromosome domain encompassing PLAG1 influence bovine stature. Nat Genet. 2011;43(5):405-13.

33. Alarid ET, Windle JJ, Whyte DB, Mellon PL. Immortalization of pituitary cells at discrete stages of development by directed oncogenesis in transgenic mice. Development. 1996;122(10):3319-29.

34. Thomas P, Mellon PL, Turgeon J, Waring DW. The L beta T2 clonal gonadotrope: a model for single cell studies of endocrine cell secretion. Endocrinology. 1996;137(7):2979-89.

35. Graham KE, Nusser KD, Low MJ. LbetaT2 gonadotroph cells secrete follicle stimulating hormone (FSH) in response to active A. J Endocrinol. 1999:162(3):R1-5.

36. Bernard DJ. Both SMAD2 and SMAD3 mediate activin-stimulated expression of the follicle-stimulating hormone beta subunit in mouse gonadotrope cells. Mol Endocrinol. 2004;18(3):606-23.

37. Rejon CA, Hancock MA, Li YN, Thompson TB, Hebert TE, Bernard DJ. Activins bind and signal via bone morphogenetic protein receptor type II (BMPR2) in immortalized gonadotrope-like cells. Cell Signal. 2013;25(12):2717-26.

38. Pierson RA, Ginther OJ. Ultrasonographic appearance of the bovine uterus during the estrous cycle. J Am Vet Med Assoc. 1987;190(8):995-1001.

39. Sirois J, Fortune JE. Ovarian follicular dynamics during the estrous cycle in heifers monitored by real-time ultrasonography. Biol Reprod. 1988:39(2):308-17.

40. Adams GP, Matteri RL, Kastelic JP, Ko JC, Ginther OJ. Association between surges of follicle-stimulating hormone and the emergence of follicular waves in heifers. J Reprod Fertil. 1992;94(1):177-88.

41. Ginther OJ, Bergfelt DR, Kulick L, Kot K. Pulsatility of systemic FSH and LH concentrations during follicular-wave development in cattle. Theriogenology. 1998;50(4):507-19.

42. Lopez H, Sartori R, Wiltbank MC. Reproductive hormones and follicular growth during development of one or multiple dominant follicles in cattle. Biol Reprod. 2005;72(4):788-95.

43. Jainudeen MR, Wahid H, Hafez E, S E. Ovulation Induction, Embryo Production and Transfer. In: Hafez B, Hafez E, S E, editors. Reproduction in farm animals. 7th ed. Oxford: Blackwell Publishing; 2000: 405-430.

44. Galloway SM, McNatty KP, Cambridge LM, Laitinen MP, Juengel JL, Jokiranta TS, et al. Mutations in an oocyte-derived growth factor gene (BMP15) cause increased ovulation rate and infertility in a dosage-sensitive manner. Nat Genet. 2000;25(3):279-83.

45. Wilson T, Wu XY, Juengel JL, Ross IK, Lumsden JM, Lord EA, et al. Highly prolific Booroola sheep have a mutation in the intracellular kinase domain of bone morphogenetic protein IB receptor (ALK-6) that is expressed in both oocytes and granulosa cells. Biol Reprod. 2001;64(4):1225-35. 
46. Mulsant $P$, Lecerf $F$, Fabre $S$, Schibler $L$, Monget $P$, Lanneluc I, et al. Mutation in bone morphogenetic protein receptor-IB is associated with increased ovulation rate in Booroola Merino ewes. Proc Natl Acad Sci U S A. 2001;98(9):5104-9.

47. Ibi T, Hirooka H, Kahi AK, Sasae Y, Sasaki Y. Genotype x environment interaction effects on carcass traits in Japanese Black cattle. J Anim Sci. 2005;83(7):1503-10.

48. Ibi T, Kahi AK, Hirooka H. Effect of carcass price fluctuations on genetic and economic evaluation of carcass traits in Japanese Black cattle. J Anim Sci. 2006;84(12):3204-11

49. Boldman KG, Kriese LA, Van Vleck LD, Kachman SD. A Manual for Use of MTDFREML. A Set of Programs to Obtain Estimates of Variances and Covariances [Draft]. USDA-ARS, Washington. DC. 1993.

50. Center for Bioinformatics and Computational Biology at University of Maryland. [ftp://ftp.ccb.jhu.edu/pub/data/assembly/Bos_taurus/ Bos_taurus_UMD_3.1/]

51. Purcell S, Neale B, Todd-Brown K, Thomas L, Ferreira MA, Bender D, et al. PLINK: a tool set for whole-genome association and population-based linkage analyses. Am J Hum Genet. 2007;81(3):559-75.

52. Barrett JC, Fry B, Maller J, Daly MJ. Haploview: analysis and visualization of LD and haplotype maps. Bioinformatics. 2005;21(2):263-5.

53. Niwa H, Yamamura K, Miyazaki J. Efficient selection for high-expression transfectants with a novel eukaryotic vector. Gene. 1991;108(2):193-9.

54. Image J. [http://imagej.nih.gov/ij/]

55. Falconer DS, Mackay TFC. Introduction to quantitative genetics. 4th ed Essex: Longman Group Ltd; 1996.

\section{Submit your next manuscript to BioMed Central and take full advantage of:}

- Convenient online submission

- Thorough peer review

- No space constraints or color figure charges

- Immediate publication on acceptance

- Inclusion in PubMed, CAS, Scopus and Google Scholar

- Research which is freely available for redistribution 